\title{
Appreciative Inquiry in der Partizipativen Gesundheitsforschung: Methodische Einblicke in eine Fallstudie im Stadtteil
}

\author{
Petra Wihofszky, Sandra Layh, Mareen Jahnke und \\ Petra Hofrichter ${ }^{1}$
}

\section{Zusammenfassung}

Appreciative Inquiry (AI) ist eine ressourcenorientierte Methode aus dem Bereich der Organisationsentwicklung und wird im anglofonen Sprachraum als eine Methode partizipativer Forschung rezipiert. AI steht für wertschätzende Erkundung: Statt an Defiziten anzusetzen, ermöglicht AI als eine partizipative Forschungsmethode, Stärken in Organisationen, Gemeinschaften oder Netzwerken zu erforschen, aufzudecken und Impulse für Veränderungen zu setzen. Im deutschsprachigen Raum ist AI als Forschungsmethode noch wenig bekannt. Im folgenden Beitrag stellen wir die Anwendung von AI als Methode Partizipativer Gesundheitsforschung exemplarisch an einer Fallstudie vor. Im Rahmen der Fallstudie untersuchten wir - eine Forschungsgemeinschaft mit Mitgliedern aus Wissenschaft und Praxis - ein Netzwerk zur Gesundheitsförderung auf Stadtteilebene. Auf der Basis der Wertehaltung von AI wurde das Design erarbeitet, Interviews mit Fachkräften des Netzwerks durchgeführt und Daten in einem gemeinsam gestalteten Prozess ausgewertet. Schlussfolgerungen aus den

1 Petra Wihofszky| Hochschule Esslingen | petra.wihofszky@hs-esslingen.de.

Sandra Layh | Hochschule Esslingen | sandra.layh@hs-esslingen.de.

Mareen Jahnke | HAG e.V.| mareen.jahnke@hag-gesundheit.de.

Petra Hofrichter | HAG e.V. | petra.hofrichter@hag-gesundheit.de.

(C) Der/die Autor(en) 2020

S. Hartung et al. (Hrsg.), Partizipative Forschung, https://doi.org/10.1007/978-3-658-30361-7_6 
Ergebnissen mündeten in die Entwicklung eines Instrumentes zur Beratung von Stadtteilen und Kommunen. Abschließend diskutieren wir in diesem Beitrag Chancen und Grenzen von AI sowie unsere verschiedenen Blickwinkel und Perspektiven innerhalb der Forschungsgemeinschaft auf den Prozess.

\section{Schlüsselbegriffe}

Appreciative Inquiry; partizipative Forschung; Gesundheitsförderung; Kommune; Beratung

\section{$1 \quad$ Einführung}

Die Ursprünge von Appreciative Inquiry (AI) liegen in der Organisations- und Teamentwicklung. In den frühen 1980er-Jahren wurde AI von Cooperrider und Kolleg*innen entwickelt, um zu untersuchen, was Organisationen Lebendigkeit gibt (Cooperrider et al. 2008). Diese Fokussierung war damals eine grundlegend neue Denkrichtung in der Organisationsentwicklung, in der zuvor immer auf Probleme und deren Behebung abgestellt worden war. Dahinter steht die Annahme, dass alle Systeme und deren Mitglieder über verborgene und ungenutzte Stärken verfügen. Werden diese aufgedeckt, stecken sie an und führen zu positiven Veränderungen.

In einem strukturierten Verfahren werden bei AI herausragende Erlebnisse und Höhepunkte, Stärken und Erfolgsfaktoren erkundet. Der sogenannte 4D-Zyklus rahmt die meisten AI-Vorhaben (Reed 2007). Die vier Ds beschreiben die Phasen: Discovery, Dream, Design, Destiny (Cooperrider et al. 2008). In Anlehnung daran charakterisieren Trajkovski, Schmied, Vickers und Jackson (2013) die Phasen des 4D-Zyklus anschaulich:

\footnotetext{
"The discovery phase (,what gives life" to the organization, that is, appreciating and valuing what is best of what is or has been), the dream phase (envisioning, what might be', affirmative exploration), the design phase (co-constructing, what should be, the ideal), and lastly, destiny (sustaining what will be, envisioned future) [...]" (S. 1225).
}

Diese Phasen sollten nicht als bloße Technik missverstanden werden - vielmehr geht es darum, diese so zu gestalten, dass sie Veränderungen in der sozialen Konstruktion der „Realität“, einen tiefgreifenden kulturellen Wandel zu erzeugen vermögen (Bushe 2007). 
Daraus leiteten wir konkret ab: In der ersten Phase Discovery geht es zunächst darum, bestehende, eventuell aber verdeckte Ressourcen und Potenziale zu erkennen, durch Erzählungen freizulegen und wertzuschätzen. In der folgenden Dream-Phase werden gemeinsam Ideen entwickelt, wie eine Zukunft aussehen könnte. Die so entstehenden Visionen werden oft als provokative Aussagen formuliert und sind frei von jeglichen gedanklichen Restriktionen. Beim Design werden diese Ideen priorisiert und es wird konkret überlegt, wie die Zukunft aussehen soll. In der Phase Destiny werden schließlich konkrete Strategien formuliert, um den Zukunftsentwurf zu erreichen (Cooperrider et al. 2008).

Wird AI als Forschungsmethode eingesetzt, so umfassen die ersten drei Phasen in der Regel das Führen wertschätzender Interviews sowie Workshops zur partizipativen Erarbeitung der Ressourcen und Potenziale und der entsprechenden Visionen. Je nach Forschungsgegenstand und -design wird der Zyklus von AI-Forschungsprojekten nicht vollständig umgesetzt: Paige und Kolleg*innen (2015) sowie Reed, Richardson, Marais und Moyle (2008) verzichten auf die Destiny-Phase, während andere diese als Teil der Forschung einbeziehen (Carter et al. 2007).

Vor allem im anglofonen Raum wird AI als Forschungsmethode nicht nur im Kontext von Unternehmen genutzt (Ludema \& Fry 2008; Reed 2007; Trajkovski et al. 2013). Im deutschsprachigen Raum ist AI bisher hauptsächlich als Verfahren zur Beteiligung von Bürger*innen bekannt, um Visionen für öffentliche Räume zu entwickeln und umzusetzen (Nanz \& Fritsche 2012). Und obwohl AI in vielen Zusammenhängen eingesetzt wird, zum Beispiel in der Teamentwicklung, der Stadtentwicklung, in Bereichen wie Schule, öffentliche Verwaltung und Hochschule, existiert kaum deutschsprachige Literatur (zur Bonsen \& Maleh 2012). Zu Forschungen mit AI ist im deutschen Sprachraum zum Zeitpunkt der Erstellung des Beitrags nichts publiziert.

In diesem Beitrag stellen wir AI als eine im deutschen Sprachraum noch wenig bekannte Forschungsmethode hier im Kontext der Partizipativen Gesundheitsforschung vor. Der Ansatz der Partizipativen Gesundheitsforschung verfolgt die koproduktive Gewinnung von Erkenntnissen mit dem Ziel, die Gesundheit und das Wohlbefinden von Menschen zu fördern und positive Veränderungen auf verschiedenen Ebenen zu bewirken (Wright 2016). Nachdem wir die Grundlagen und die besondere Wertehaltung von AI in der Einleitung erläuterten, führen wir im zweiten Abschnitt in unsere Fallstudie und im dritten Abschnitt in die Forschungsgemeinschaft ein. Im Zentrum des Beitrags steht im vierten Abschnitt der Prozess, wie wir AI in unserer Fallstudie umsetzten und welche Impulse für Veränderungen wir aus den Ergebnissen ableiteten. Abschließend diskutieren wir im fünften Abschnitt die Methode AI unter Berücksichtigung unterschiedlicher Perspektiven. Im sechsten Abschnitt geben wir einen Ausblick, welche Rolle AI in der Partizipativen Gesundheitsforschung spielen könnte. 


\section{$2 \quad$ Kontext der Fallstudie}

Gesundheit stellt sich im Alltag des Menschen in der Interaktion mit seiner Umwelt und seinen Lebensbedingungen her. Nach der Ottawa-Charta der Weltgesundheitsorganisation (WHO) wird Gesundheit dort geschaffen, „wo Menschen spielen, lernen, arbeiten und leben“ (WHO 1986) - in den Lebenswelten oder Settings.

In kommunalen Settings wie Stadtteilen und Quartieren wird Gesundheitsförderung nach dem Modell integrierter kommunaler Strategien (IKS) umgesetzt (Böhme \& Reimann 2018). ${ }^{2}$ Auf einen Nenner gebracht, ist das Ziel einer IKS, „,von einem Nebeneinander [...] zu einem Miteinander" der Akteur*innen in der Gesundheitsförderung zu gelangen (Richter-Kornweitz \& Utermark 2013, S. 14). Der gemeinsame Bezugspunkt in der Zusammenarbeit ist die Bewohnerschaft und das Bestreben, Bedingungen zu schaffen, die das Wohlbefinden und die Lebensqualität der Menschen in ihrem Wohnumfeld fördern. Eine IKS aufzubauen gilt als höchst komplex. Sie stellt große Anforderungen an die Organisation der Zusammenarbeit. Die zentrale Herausforderung besteht darin, Fachkräfte aus dem Gesundheits-, Bildungs- und sozialen Sektor mit ihren unterschiedlichen beruflichen Rollen, $\mathrm{Zu}$ ständigkeiten und Aufgabenfeldern zusammenzubringen und gesundheitsfördernde Maßnahmen zu vernetzen. Seit 2012 wird im Hamburger Stadtteil Rothenburgsort eine IKS aufgebaut. Der Prozess zum Aufbau einer IKS wurde von der Koordinierungsstelle Gesundheitliche Chancengleichheit Hamburg (KGC) initiiert und seitdem fachlich begleitet. ${ }^{3}$ Rothenburgsort ist einer der ärmsten Stadtteile Hamburgs. Knapp die Hälfte aller Kinder wächst in Bedarfsgemeinschaften, ein Drittel in Ein-Eltern-Familien auf - die Inanspruchnahme von Maßnahmen für Hilfen zur Erziehung ist hoch. Charakteristisch für den kleinen Stadtteil von knapp 10.000 Einwohner*innen sind die kulturelle Vielfalt, die Insellage (begrenzt durch die Flüsse Elbe und Bille, große Verkehrsachsen sowie Industrie- und Gewerbegebiete) und eine hohe Anzahl von Akteur*innen vor Ort, die sich für die Gesundheit von Kindern und Familien einsetzen. Viele junge Menschen bis 18 Jahre haben einen

2 Für eine ausführliche Darstellung verweisen wir auf Publikationen des Kooperationsverbundes Gesundheitliche Chancengleichheit (https://www.gesundheitlichechancengleichheit.de).

3 Die Koordinierungsstelle Gesundheitliche Chancengleichheit Hamburg (KGC) ist an der Hamburgischen Arbeitsgemeinschaft für Gesundheitsförderung e. V. (HAG) angesiedelt. Die KGC fungiert als Fachstelle für Akteur*innen der Gesundheitsförderung für und mit Menschen in belasteten Lebenslagen. Rothenburgsort wurde als Modellstandort für eine IKS im Rahmen der landesweiten Präventionsstrategie „Pakt für Prävention“ ausgewählt und wird von der Behörde für Gesundheit und Verbraucherschutz (BGV) gefördert und unterstützt. 
Migrationshintergrund (78,6\%, im Vergleich 51,3\% hamburgweit) (Statistisches Amt für Hamburg und Schleswig-Holstein 2018a, 2018b).

Akteur*innen aus den unterschiedlichen Einrichtungen Rothenburgsorts begannen im Rahmen einer dreitägigen Fortbildungsreihe mit dem strukturierten Aufbau einer IKS. Die Workshops zielten auf die Entwicklung einer gemeinsamen Arbeitshaltung. Nachdem die Akteur*innen gemeinsam Ziele und Handlungsleitlinien erarbeitet hatten, formierte sich das Netzwerk "Gesund aufwachsen in Rothenburgsort!“. Als Leitziel formulierten die Teilnehmer*innen des Netzwerks, über den Aufbau einer IKS positive Entwicklungsbedingungen für Kinder im Alter von 0-10 und deren Familien fördern zu wollen. Die IKS in Rothenburgsort ist seitdem darauf ausgerichtet, Unterstützungsangebote für Kinder, Jugendliche und deren Familien aus den Bereichen Gesundheit, Bildung, Soziale Arbeit und Bewegung aufeinander abzustimmen. Ein Schwerpunkt ist dabei die gesundheitsgerechte Gestaltung von Übergängen zwischen den Lebenswelten, beispielsweise vom Elternhaus in eine Kita oder von dort in die Grundschule.

Dieser Aufbauprozess der IKS in Rothenburgsort war eingebettet in hamburgund bundesweite Strategien, Programme und Projekte. Rothenburgsort war somit nicht alleine mit dem Ausbau einer IKS beschäftigt, sondern Teil eines bundesweiten Lernprozesses. Dazu gehörte und gehört auch die Beteiligung am Forschungsprojekt KEG. KEG steht für „Kommunale Entwicklung von Gesundheitsstrategien“ und gehört zum Forschungsverbund PartKommPlus, der vom Bundesministerium für Bildung und Forschung gefördert wird (http://partkommplus.de). Ziel von KEG ist die (Weiter-)Entwicklung von IKS. In einer Fallstudie untersuchten wir - die Hamburgische Arbeitsgemeinschaft für Gesundheitsförderung e. V. (HAG), die Behörde für Gesundheit und Verbraucherschutz (BGV), der Bezirk Hamburg-Mitte und die Hochschule Esslingen - in einer Forschungsgemeinschaft die Entwicklung der IKS und die Zusammenarbeit im Netzwerk am Beispiel von Rothenburgsort. Zu Beginn des Forschungsprojekts befand sich das Netzwerk jedoch in einer Krise: Die Zusammenarbeit der lokalen Akteur*innen war aufgrund vielfältiger Veränderungen ins Stocken geraten. Um Parallelstrukturen zu vermeiden, personelle und institutionelle Ressourcen zu schonen sowie die Netzwerkarbeit zu redynamisieren, wurde das Netzwerk einvernehmlich mit allen Beteiligten in die Regionalkonferenz Rothenburgsort - ein Zusammenschluss der sozialen Einrichtungen vor Ort - integriert (Göldner \& Hofrichter 2019).

Der Forschungsverbund PartKommPlus, und somit auch KEG, arbeitet, wie bereits erwähnt, mit dem Ansatz der Partizipativen Gesundheitsforschung. 


\section{Beschreibung der Forschungsgemeinschaft}

In der Partizipativen Gesundheitsforschung definiert sich Forschungsgemeinschaft als Zusammenschluss verschiedener Interessengruppen, wie beispielsweise Bürger*innen, Vertreter*innen der Zivilgesellschaft, Fachkräfte aus dem Gesundheits-, Sozial- oder Bildungswesen, Wissenschaftler ${ }^{\star}$ innen und andere, die zusammenkommen, um einen Forschungsprozess gemeinsam zu gestalten und zu steuern. Als Forscher*in werden dabei nicht nur die beteiligten Wissenschaftler*innen bezeichnet, sondern alle Mitglieder der Forschungsgemeinschaft (von Unger 2014a; Wright 2013). Nach Bergold und Thomas (2012) soll in Forschungsgemeinschaften

„die Differenz zwischen akademischer Weltsicht und der Weltsicht der Forschungspartner/innen [...] für den Erkenntnisprozess fruchtbar werden. Es geht also um gegenseitige Neugier auf das, was die andere Seite kann und weiß und was man von ihr lernen kann. Damit erhalten alle Beteiligten neue Rollen und Aufgaben, die sich deutlich von denjenigen der ,klassischen` Forschung unterscheiden“" (S. 13).

In KEG bildete sich der Kern der Forschungsgemeinschaft bereits während der Phase der Antragsstellung. Gemeinsam entwickelten die HAG und die Hochschule als primäre Partnerinnen im fachlichen Austausch mit der BGV den Fokus für die Fallstudie. Die Absicht war es, die Anliegen der Bewohnerschaft kennenzulernen und einzubeziehen, den Auf- und Ausbau einer IKS in seinen Schritten, Dynamiken, förderlichen und hemmenden Bedingungen zu verstehen und Empfehlungen für Hamburg zu generieren.

In der Anfangsphase forderten wir alle Mitglieder des Netzwerks „Gesund aufwachsen in Rothenburgsort!“auf, in der Forschungsgemeinschaft mitzuwirken. Im Unterschied zu herkömmlichen Forschungsprojekten sollten in KEG auch Fachkräfte, Akteur*innen des Stadtteils und Bewohner*innen als Mitforschende gewonnen werden. Die Forschungsgemeinschaft sollte ein Forum für einen Dialog oder ein sogenannter kommunikativer Raum (Bergold \& Thomas 2012) sein, in dem sich in interaktiven Prozessen des Perspektivenaustauschs und -verhandelns neue Erkenntnisse entwickeln und Wirkungen entstehen (Springett et al. 2011; Tuckermann \& Rüegg-Stürm 2010). Die Stärke einer solchen Forschungsgemeinschaft liegt im Kontext unserer Fallstudie darin, Interessen aus dem Stadtteil (Bottom-up) und kommunales Handeln (Top-down) als nebeneinanderlaufende Prozesse wahrzunehmen und aufeinander zu beziehen (Laverack \& Labonte 2000). Wir bezeichnen diesen Ansatz als einen mittleren Weg zwischen Top-down und Bottom-up (Wihofszky 2015). Konkret bedeutet dies, zum Beispiel einerseits kommunale Gesundheits- und Sozialdaten zu nutzen, andererseits auch Bewohner*innen dazu zu motivieren, mitzuforschen und sich einzubringen. 
Wie wir bereits beschrieben haben, fusionierte das Netzwerk „Gesund aufwachsen in Rothenburgsort!" mit der bestehenden Regionalkonferenz. Dies führte dazu, unseren Ansatz der Forschungsgemeinschaft zu modifizieren. Während unseres Prozesses waren Mitarbeitende der HAG, der BGV, des Bezirksamtes Hamburg-Mitte, darunter auch die Koordinatorin des Netzwerks, und Wissenschaftlerinnen der Hochschule beteiligt. Weitere Akteur*innen des Netzwerks konnten für eine Beteiligung an der Forschungsgemeinschaft nicht gewonnen werden, da sich ihre Tätigkeitsfelder, ihre Prioritäten und ihre Beteiligung in der Netzwerkarbeit in dieser Zeit veränderten. Aktuell besteht der Kern der Forschungsgemeinschaft aus drei Mitarbeiterinnen der HAG und zwei Wissenschaftlerinnen der Hochschule Esslingen.

Die Forschungsgemeinschaft bildete während des AI-Prozesses den Dreh- und Angelpunkt und traf sich in regelmäßigen Abständen - in ihr wurden gemeinsam Entscheidungen getroffen und Forschungsschritte geplant und umgesetzt (Cook et al. 2018). Wir trafen dennoch auch Absprachen zu einem arbeitsteiligen Vorgehen. So übernahmen die Wissenschaftlerinnen die Aufgaben, die beteiligten Partnerinnen methodisch darin zu schulen, Erhebungen mittels AI-Interviews durchzuführen und die erhobenen Daten für die partizipative Auswertung aufzubereiten. Wiederum lag es in der Zuständigkeit der HAG die zusätzlichen mitforschenden Bewohnerinnen aus dem Stadtteil in gesundheits- und gesundheitsförderungsbezogenen Inhalten und Konzepten zu schulen, Kontakt ins Forschungsfeld und zu Interviewpartner*innen herzustellen, in Bezirksausschüssen und Stadtteileinrichtungen für das Projekt und den mit dem Projekt verbundenen lokalen Nutzen zu sensibilisieren und die Erhebungen im Netzwerk zu realisieren. Die Auswertung und Formulierung von Empfehlungen sowie der Transfer in die Praxis wiederum waren gemeinsam in der Forschungsgemeinschaft ausgehandelte und durchgeführte Schritte.

Im Laufe des AI-Prozesses konnten zusätzlich zu den Forschenden der Forschungsgemeinschaft Bewohnerinnen des Stadtteils als Mitforschende gewonnen werden. Diese erfragten mit der Methode AI gesundheitliche Anliegen und Interessen der Bewohnerinnen Rothenburgsorts, speziell von Familien mit Kindern unter zehn Jahren. Das Engagement der Bewohnerinnen, die sich später auch an Treffen der Forschungsgemeinschaft beteiligten, ergab sich erst im Laufe des Prozesses. Eine Honorarkraft aus einer Institution des Stadtteils, die sich ursprünglich als Mitforschende für die Netzwerkbefragung beteiligen wollte, identifizierte sich stärker mit der Sicht der Bewohner*innen und sah sich als Vertreterin dieser. So wurde gemeinsam beschlossen, diese unerwartete Entwicklung und deren Potenzial zu nutzen und neben der Netzwerkbefragung auch die Anliegen und Perspektiven der Bewohner*innen mit AI zu erkunden. 
In der Darstellung des AI-Prozesses im Rahmen dieses Beitrags beziehen wir uns ausschließlich auf die Netzwerkbefragung, da sie den Schwerpunkt unserer Forschung bildet. Die Ergebnisse aus der Bewohner*innenbefragung flossen aber in den Praxistransfer ein, den wir in Abschnitt 4.3.4 thematisieren.

\section{$4 \quad$ Methodisches Vorgehen}

In Rothenburgsort begann der Aufbau einer IKS in einer Aufbruchsstimmung. Die Bereitschaft der Fachkräfte, sich zu beteiligen, ließ aber nach, und wir stellten uns als Forschungsgemeinschaft die Frage, wie das Netzwerk redynamisiert werden kann. Für unsere Untersuchung wählten wir die Methode Appreciative Inquiry (AI) - übersetzt: wertschätzende Erkundung (zur Bonsen \& Maleh 2012). Die Methode erschien uns geeignet, da wir nach Impulsen für die Wiederbelebung des Netzwerks in Rothenburgsort suchten. Die Forschungsgemeinschaft bildete den Dreh- und Angelpunkt im methodischen Vorgehen von AI, das, wie wir bereits darstellten, die vier Phasen des 4D-Zyklus (Discovery, Dream, Design und Destiny) durchläuft (Cooperrider et al. 2008). Im Folgenden stellen wir unser Vorgehen vor, das wir in Vorbereitung (4.1), Erhebung (4.2) und Auswertung und Transfer in die Praxis (4.3) untergliedern.

\subsection{Vorbereitung}

\subsubsection{Passung der Forschungsfrage}

KEG verfolgt, wie alle partizipativen (Gesundheits-)Forschungsprojekte, zwei miteinander verbundene Zielebenen. Es sollen Veränderungen initiiert und vorangebracht sowie gleichzeitig Erkenntnisse darüber gewonnen werden, was diese Veränderungen ermöglicht (von Unger 2014a). Dabei geht es nicht um Forschung über, sondern mit Menschen (Cook 2012). In KEG beschloss die Forschungsgemeinschaft, die Zusammenarbeit im Rahmen der IKS in Rothenburgsort dialogisch zu untersuchen. Die Forschungsgemeinschaft stellte sich die Frage, wie die nach der Fusion neue Konstellation der Zusammenarbeit (wieder) funktionieren könnte. Sie übersetzte dieses Interesse in die handlungsleitende Frage: Wie kann die IKS im Stadtteil (wieder) in Schwung gebracht werden? Auf der wissenschaftlichen Erkenntnisebene stellte sich die Frage, wie im Rahmen solcher kommunalen Strategien erfolgreich zusammengearbeitet werden kann, was hierfür förderliche und was hinderliche Faktoren sind. Beide Erkenntnisebenen verschränken sich 
miteinander und zielen auf das in der Forschungsgemeinschaft geteilte Interesse $\mathrm{ab}$, einen Beitrag zu Veränderungen in der Praxis zu leisten.

AI ist, wie wir bereits darstellten, eine Methode, die ursprünglich aus der Organisationsentwicklung stammt (Cooperrider et al. 2008). Wertschätzung bedeutet in der Methode, dass sie an guten Erfahrungen in Organisationen bzw. der Zusammenarbeit von Menschen ansetzt. Da die zu untersuchende Netzwerkarbeit eine Form der organisierten Zusammenarbeit darstellt, eignete sich die Anwendung von AI für unseren Forschungskontext. Die Methode fördert das Erzählen positiver Geschichten, um vorhandene Ressourcen und Potenziale einer Organisation aufzudecken. Die Zusammenarbeit in Rothenburgsort hatte bereits gut funktioniert. Sie nun wiederzubeleben, bedurfte der Kenntnis der Faktoren, die zum damaligen Erfolg der Zusammenarbeit beigetragen hatten. Sie sollten gestärkt werden, um die Zusammenarbeit erneut zu fördern. Wir fragten deshalb nicht nach Defiziten und Fehlern, die gemacht worden waren, sondern danach, was in der Netzwerkarbeit positiv erlebt worden war und welche Erwartungen die Befragten zum Zeitpunkt der Untersuchung an die Netzwerkarbeit stellten.

Ein wichtiger Bestandteil von AI ist zudem die Formulierung von Wünschen und Visionen für die Zukunft der Organisation bzw. der Zusammenarbeit (zur Bonsen \& Maleh 2012). Mit dem Einsatz der Methode soll aber auch ein Veränderungsprozess in Richtung dieser Wünsche und Visionen initiiert werden. Eine solche Veränderung war auch ein Ziel für das fusionierte Netzwerk. Da partizipative Forschung prinzipiell auf Veränderung abzielt, passte AI als Forschungsmethode (Reed 2007). Die Stärken von AI decken sich mit den Ansätzen Partizipativer Gesundheitsforschung, die ebenfalls ressourcen-, empowerment- und dialogisch orientiert sind (Reed 2007; von Unger 2014a).

\subsubsection{Finden von Kernthemen}

Entsprechend der Discovery-Phase bereiteten wir in der Forschungsgemeinschaft den AI-Prozess im Rahmen von zwei Schulungsworkshops vor. Die Workshops wurden von Hochschulseite konzipiert und moderiert. Wir thematisierten die methodologischen Grundlagen, wie den 4D-Zyklus, sowie die wertschätzende Haltung, die AI als Methode auszeichnet. Als ein wesentlicher Schritt werden in der Discovery-Phase sogenannte Key Themes (Kernthemen) erarbeitet, die in die weitere Befragung mit einfließen (Reed 2007; zur Bonsen \& Maleh 2012). Kernthemen definieren sich als diejenigen Themen, die einer Gruppe für die Zukunft überaus wichtig sind. Nach Reed (2007, S. 70) sollten Kernthemen darüber hinaus für möglichst viele am Prozess Teilnehmende relevant sein (inclusivity) und die Suche nach positiven Aspekten für zukünftige Veränderungen ermöglichen (focus on the positive). Die Kernthemen, die in dieser Phase identifiziert werden, weisen bereits in die Richtung, in die sich 
der Prozess entwickeln kann. Dass das Finden von Kernthemen kein einfacher Schritt in der Discovery-Phase ist, stellen Trajkovski et al. (2013) fest:

\begin{abstract}
"The choice of an affirmative topic may appear to be an easy task. However, in practice, this may create difficulties for a novice AI researcher, as the starting point for most research begins with identifying and framing a problem. AI requires the researcher to move away from the traditional problem orientation to an appreciative approach" (S. 1225-1226).
\end{abstract}

Um passende Kernthemen herauszufinden, befragten sich im Rahmen des ersten Schulungsworkshops je zwei Mitglieder der Forschungsgemeinschaft gegenseitig in einem Interview. Die Hochschule nahm an der Übung nicht teil, da sie diesen methodischen Prozess moderierte und begleitete. Als Grundlage verwendeten wir den von zur Bonsen und Maleh (2012) vorgeschlagenen Basisleitfaden. Der Basisleitfaden umfasst drei Themenblöcke: Fragen dazu, wie die Organisation erlebt wird, einen Themenblock mit Fragen zum Finden der Kernthemen und Fragen, wie sich die am Prozess Beteiligten die Zukunft ihrer Organisation vorstellen. Wir passten den Basisleitfaden an die Fallstudie an - statt auf ein Unternehmen oder eine Organisation zu fokussieren, wurde nach dem Netzwerk, das die IKS in Rothenburgsort aufgebaut hatte, gefragt. Mit diesem Vorgehen wollten wir Erzählungen generieren, aus denen sich die Kernthemen herausarbeiten ließen. Mit den Interviews intendierten wir auch, die für einen AI-Prozess wertschätzende Haltung sowie das erzählgenerierende Fragen in der qualitativen Forschung einzuüben und eine selbstreflexive Forschungsperspektive einzunehmen (Helfferich 2011; von Unger 2014b).

Die Inhalte der Interviews dokumentierten wir mit Moderationskarten an einer Pinnwand und nutzten die Ergebnisse, um mögliche Kernthemen zu identifizieren. Davon ausgehend einigte sich die Forschungsgemeinschaft auf ein einziges Kernthema als Schwerpunkt der AI-Befragung: „Der Neustart der IKS in Rothenburgsort nach der Fusionierung des Netzwerks“.

\title{
4.1.3 Entwicklung des Interviewleitfadens
}

Wie Interviews auf der Wertehaltung von AI geführt werden können, geht auf die Begründer*innen von AI zurück, wird aber von Projekten und Forschungsteams für den jeweiligen Kontext adaptiert. Quaintance, Arnold und Thompson (2010) beispielsweise verwendeten neun erzählgenerierende Fragen, um die Ärzt*innen-Ausbildung weiterzuentwickeln. Die Fragen zielten auf die Genese von Geschichten über erlebte Professionalität. Scerri, Innes und Scerri (2015) stellten acht Fragen, um positiv erlebte Erfahrungen in der Demenzversorgung eines geriatrischen Krankenhauses zu erheben und hieraus Qualitätsfaktoren abzuleiten. In einer 
Studie arbeiteten Reed, Pearson, Douglas, Swinburne und Wilding (2002) mit AI-Interviews, um die Entlasspraxis aus Krankenhäusern zu untersuchen und weiterzuentwickeln. Salyers, Firmin, Gearhart, Avery und Frankel (2015) erhoben durch Mitforschende Narrative, um Gelingensfaktoren für die Arbeit in psychiatrischen Einrichtungen zu identifizieren, verwendeten hierfür aber lediglich zwei Fragen - eine nach einer positiven Erfahrung und eine nach der idealen Zukunft der psychiatrischen Einrichtung.

In KEG orientierten wir uns an dem von zur Bonsen und Maleh (2012) vorgeschlagenen Basisleitfaden mit einer Einteilung in drei Themenblöcken. Wie wir bereits in Abschnitt 4.1.2 darstellten, diente uns der Basisleitfaden auch dazu, die Kernthemen zu finden. Die Themen und Erzählaufforderungen passten wir an und nahmen Fragen zum Kernthema „Der Neustart der IKS in Rothenburgsort nach der Fusionierung des Netzwerks" darin auf. Die drei Themenblöcke des Interviewleitfadens entsprachen den ersten beiden Phasen Discovery und Dream des 4D-Zyklus. Diese beiden Phasen in einer Erhebung zusammenzufassen, ist ein übliches Vorgehen in AI-Forschungsprojekten (Trajkovski et al. 2013). In dem von uns adaptierten Interviewleitfaden stellte es sich folgendermaßen dar:

Im Sinne der Discovery-Phase befasste sich der erste Themenblock mit der Wahrnehmung des Netzwerks zum Aufbau der IKS. Er umfasste speziell die Anfangszeit des Netzwerks, denn diese wurde von den Mitgliedern der Forschungsgemeinschaft, die diese Phase vor Ort auch selbst erlebt hatten, als überaus gelungen erinnert. Außerdem sollte in diesem Themenblock von einer besonders positiven Erfahrung im Netzwerk erzählt, der Nutzen des Netzwerks auf verschiedenen Ebenen beleuchtet und über belebende Faktoren gesprochen werden.

Der zweite Themenblock war ebenfalls ein Teil der Discovery-Phase und umfasste das Kernthema Zusammenarbeit im fusionierten Netzwerk. Die ganz persönliche Sicht auf die nun neue Zusammenarbeit und was dabei wichtig war sowie Ideen für eine gelingende Gestaltung dieser wurden hier thematisiert.

Im dritten Block ging das Interview in die Dream-Phase über. Es wurde angeregt, eine mögliche Zukunft für das Netzwerk zu entwerfen. Der Themenblock umfasste eine Frage nach dem Idealzustand und ein Austausch über die Ziele, die sich das Netzwerk aus Sicht der Befragten vornehmen sollte. Hier wurde bereits die dritte Phase Design des 4D-Zyklus vorbereitet.

In Tab. 1 sind die drei Themenblöcke, die dazugehörigen Themen sowie eine der vorgeschlagenen Erzählaufforderungen aufgelistet. 
Tab. 1 Interviewleitfaden in Anlehnung an zur Bonsen \& Maleh (2012)

\begin{tabular}{|c|c|}
\hline Themenblock & Thema und einleitende Erzählaufforderung \\
\hline \multirow[t]{4}{*}{$\begin{array}{l}\text { Wahrnehmung } \\
\text { des Netzwerks }\end{array}$} & $\begin{array}{l}\text { Anfangszeit des Netzwerks } \\
\text { Lassen Sie uns mit der Anfangszeit (2012/13) des Netzwerks zum } \\
\text { Aufbau der IKS beginnen. Falls Sie nicht von Beginn dabei waren, } \\
\text { so lassen Sie uns über Ihre Anfangszeit im Netzwerk sprechen. Ich } \\
\text { möchte Sie zunächst bitten, mir zu erzählen, wie und wann Sie zum } \\
\text { Netzwerk gekommen sind. Etc. }\end{array}$ \\
\hline & $\begin{array}{l}\text { Eine für Sie besonders positive Erfahrung } \\
\text { Während der Arbeit des Netzwerks zum Aufbau einer IKS gab es } \\
\text { wahrscheinlich Höhen und Tiefen. Ich möchte Sie bitten, sich nun an } \\
\text { ein Ereignis oder eine Phase zu erinnern, die für Sie ein Höhepunkt } \\
\text { war: eine Zeit, in der Sie begeistert waren, in der Sie sich motiviert } \\
\text { gefühlt haben, in der Sie sich vielleicht besonders einbringen und } \\
\text { etwas bewirken konnten. Erzählen Sie mir bitte von dieser positiven } \\
\text { Erfahrung oder Phase: Was genau haben Sie erlebt? Etc. }\end{array}$ \\
\hline & $\begin{array}{l}\text { Was Sie an Ihrem Engagement und Ihrer Person besonders schätzen } \\
\text { Lassen Sie uns jetzt über einige Dinge sprechen, die Sie in Bezug auf } \\
\text { sich selbst und auf den Aufbau des Netzwerks und der IKS am meis- } \\
\text { ten schätzen. Ohne bescheiden zu sein, was schätzen Sie an Ihrem } \\
\text { eigenen Engagement im Netzwerk zum Aufbau der IKS am meisten? } \\
\text { Etc. }\end{array}$ \\
\hline & $\begin{array}{l}\text { Belebende Faktoren } \\
\text { Sie haben mir ja bereits von einer besonders positiven Erfahrung im } \\
\text { Netzwerk und darüber, was diese Erfahrung möglich machte, erzählt. } \\
\text { Lassen Sie uns nun darüber sprechen, welche Faktoren es sind, die } \\
\text { dem Netzwerk im Allgemeinen Lebendigkeit und Stärke geben. Was } \\
\text { sind, Ihrem Erleben oder Ihren Beobachtungen nach, belebende } \\
\text { Faktoren? Etc. }\end{array}$ \\
\hline Das Kernthema & $\begin{array}{l}\text { Belebung der Zusammenarbeit im Rahmen der IKS und nach der } \\
\text { Zusammenlegung des Netzwerks } \\
\text { Für eine bedarfsorientierte Abstimmung gesundheitsfördernder An- } \\
\text { gebote für Kinder, Jugendliche und ihre Familien im Stadtteil ist die } \\
\text { Zusammenarbeit aller Akteur*innen wichtig. Bis vor ca. einem Jahr } \\
\text { war das Netzwerk das Gremium dafür. Zur Entlastung aller wurde } \\
\text { das Netzwerk in die Regionalkonferenz Rothenburgsort integriert. } \\
\text { Wie kann diese Zusammenarbeit aus Ihrer Sicht lebendig gehalten } \\
\text { bzw. gestaltet werden? Etc. }\end{array}$ \\
\hline
\end{tabular}




\begin{tabular}{|c|c|}
\hline Themenblock & Thema und einleitende Erzählaufforderung \\
\hline \multirow[t]{2}{*}{ Die Zukunft } & $\begin{array}{l}\text { Wenn alles so ist, wie es sein soll ... } \\
\text { Stellen Sie sich vor, Sie wachen morgens auf und alles ist so, wie es } \\
\text { sein soll: Die IKS in Rothenburgsort funktioniert und ist sehr erfolg- } \\
\text { reich. Die Menschen im Stadtteil, die Mitglieder des Netzwerks sowie } \\
\text { weitere Beteiligte sind begeistert. Welche Dinge sind dafür über Nacht } \\
\text { verändert worden? }\end{array}$ \\
\hline & $\begin{array}{l}\text { Ziele } \\
\text { Was sollte aus Ihrer Sicht mit der IKS erreicht werden (für die Bewoh- } \\
\text { nerinnen des Stadtteils, für die Akteur*innen, für die Gesellschaft im } \\
\text { Allgemeinen etc.)? Wie müsste sich das Netzwerk entwickeln, damit } \\
\text { Sie sich auch weiterhin gerne darin engagieren? }\end{array}$ \\
\hline
\end{tabular}

Die Mitglieder der Forschungsgemeinschaft pretesteten den Leitfaden in ihrem Arbeitsumfeld. Im Pretest erwies es sich als schwierig, die Fragen in ihrem Wortlaut zu stellen. Deshalb wurde vereinbart, den Leitfaden als Hintergrundfolie oder Gedächtnisstütze zu nutzen, aber in der Formulierung der Erzählaufforderungen und möglicher Detaillierungsfragen eine flexible, offene und dialogische Handhabung $\mathrm{zu}$ ermöglichen, wie es auch in anderen qualitativen Interviewformen umgesetzt wird (Helfferich 2011).

\subsection{Erhebung}

\subsubsection{Stichprobe und Feldzugang}

Die Zusammensetzung der Stichprobe wurde in der Forschungsgemeinschaft abgestimmt. Aktuelle und auch ehemalige Mitglieder des Netzwerks sollten als Interviewpartner*innen ausgewählt werden. Die Forschungsgemeinschaft fasste den Entschluss, auch nicht mehr beteiligte Personen zu befragen. Durch Interviews mit Personen, die diese Phase erlebt hatten, aber zum Zeitpunkt der Forschung nicht mehr beteiligt waren, sollten geeignete Anknüpfungspunkte aus der Anfangsphase des Netzwerks herausgefiltert werden. Hier wurden die Vorschläge der HAG und des Bezirks berücksichtigt, da sie die Feldkompetenz für die Auswahl passender Interviewpartner*innen mitbrachten.

Zugang zu den zu Befragenden hatten die Mitglieder der Forschungsgemeinschaft durch ihre Berufsrolle. Ein Mitglied der Forschungsgemeinschaft war selbst Teil des Netzwerks. Sie kündigte die Befragung in einem Netzwerktreffen an und erläuterte dort die Ausrichtung der Untersuchung auf den Neustart der Gesundheitsförderungsaktivitäten. Mitglieder der Forschungsgemeinschaft nahmen zudem persönlichen 
und/oder telefonischen Kontakt zu den potenziellen Interviewpartner*innen auf. Diesen Part gestalteten die Hamburger Partnerinnen der Forschungsgemeinschaft.

So wurden neun Personen aus sieben verschiedenen Institutionen dafür gewonnen, sich an der Befragung zu beteiligen. Insgesamt waren 15 Personen angefragt worden, von denen sechs aus verschiedenen Gründen - Zeitmangel, Personalausfall, Mutterschutz etc. - absagten. Die Stichprobe umfasste zwei ehemalige Netzwerkkoordinatorinnen, fünf Akteurinnen von Einrichtungen im Stadtteil und zwei Mitarbeiterinnen der Bezirksverwaltung.

\subsubsection{Ethik und Datenschutz}

Für den Datenschutz sowie ethische Aspekte wurden verschiedene Maßnahmen von der Forschungsgemeinschaft initiiert. Die Wissenschaftlerinnen der Hochschule entwarfen ein Informationsschreiben über das Projekt, dessen Ziele, die zu erhebenden Daten sowie das Vorgehen im Forschungsprozess. Das Schreiben wurde in der Forschungsgemeinschaft beschlossen und anschließend allen Interviewpartner*innen im Vorfeld des Interviews zur Verfügung gestellt. Unmittelbar vor dem Interview wurden offene Fragen im Gespräch geklärt (von Unger 2014a) und das informierte Einverständnis schriftlich eingeholt (Helfferich 2011).

Nachdem wir in der Forschungsgemeinschaft entschieden hatten, ein Video über das Projekt und die Ergebnisse der AI-Befragung zu produzieren, holten wir ein zusätzliches Einverständnis ein. In dem Video wurden Filmaufnahmen aus dem Forschungsprozess sowie Fotos der Beteiligten und O-Töne aus den Interviews verwendet. Das Video sollte über den Projektzeitraum hinaus genutzt werden können und ein Medium des Feedbacks in und der Wertschätzung für den Stadtteil und dessen Akteurinnen sein. Mit der Entscheidung für ein Video gaben wir dem Empowerment den Vorzug vor der umfassenden Anonymisierung (von Unger 2014a; von Unger \& Narimani 2012). Da wir, wie auch Narimani (2014), das informierte Einverständnis als forschungsbegleitenden Prozess verstehen, waren die Mitglieder des Netzwerks, neben uns selbst, die ersten Personen, die das Video sahen und die Möglichkeit erhielten, Änderungswünsche zu nennen. Es stieß durchweg auf positive Resonanz. ${ }^{4}$

Ein Ethikvotum, wie es mittlerweile auch im sozialwissenschaftlichen Bereich vermehrt beantragt wird (von Unger 2014b), wurde erst in der zweiten Förderphase des Projekts eingeholt.

4 Das Video kann über die Homepage des Forschungsverbundes PartKommPlus angesehen werden: http://partkommplus.de/teilprojekte/keg/gesund-aufwachsen-in-rothenburgsort/. 


\subsubsection{Durchführung der Al-Interviews}

Die Erhebungsphase erstreckte sich über zwei Monate. Die Länge der neun geführten Interviews variierte zwischen 20 und 70 Minuten und lag im Durchschnitt bei ca. 40 Minuten. Die Interviews wurden ausschließlich von den Hamburger Partnerinnen der Forschungsgemeinschaft geführt. Sieben Interviews fanden in den Büroräumen der jeweiligen Befragten statt, zwei in den Räumen der Interviewenden. Für den Zugang wurden die beruflichen Kontakte genutzt. Termine wurden per E-Mail und am Telefon vereinbart.

Direkt in bzw. nach der Interviewsituation füllten die Mitforschenden zu jedem der geführten Interviews Datenbögen aus und fertigten Memos an. Für beides wurden von den Wissenschaftlerinnen Vorlagen zur Verfügung gestellt. Die Forschungsgemeinschaft nutzte die Datenbögen und Memos für eine erste Rückmeldung über den Stand und Verlauf der Erhebungen. Zudem wurde beides als Einstieg in die partizipative Auswertung der einzelnen Interviews verwendet - die Dokumente fungierten als Gedankenstütze.

Alle Mitforschenden nahmen die Interviews mit Sprachaufnahmegeräten auf und stellten diese verschlüsselt dem Hochschulteam zur Aufbereitung für die Auswertung zur Verfügung.

\subsection{Auswertung und Transfer der Ergebnisse}

Über eine systematische Auswertung von AI-Interviews finden sich in der Fachliteratur wenige Anhaltspunkte. Die Auswertungsstrategie wird in der Regel dem Forschungskontext angepasst. So führten zum Beispiel in einer Studie von Quaintance, Arnold und Thompson (2010) Medizin-Studierende Interviews mit Lehrenden über Professionalität. Die Studierenden schrieben Narrative über die erfahrenen Geschichten und reflektierten diese. In einem iterativen Prozess, bestehend aus offenem Codieren und anschließendem Diskutieren, analysierten die Wissenschaftler ${ }^{\star}$ innen die Narrative und Reflexionen der Studierenden. Ein induktives Vorgehen wählten Dematteo und Reeves (2011). Zunächst analysierten sie das Interviewmaterial, um Schlüsselthemen in interprofessionellen Initiativen herauszufinden. Anschließend wurden die Analyseergebnisse in der Forschungsgruppe diskutiert und abgestimmt. In einer für einen AI-Prozess typischen Form erfolgte die Auswertung in der Studie von Scerri und Kollegæinnen (2015): Nach gegenseitigen Interviews sammelten die Beteiligten Themen, die in den Geschichten relevant waren, um darauf basierend eine Zukunftsvision sowie Vorschläge und Umsetzungspläne für eine Demenzversorgung zu erarbeiten. Ein ähnliches Vorgehen findet sich auch in weiteren Studien (Carter et al. 2007; Farrell et al. 2003). 
Wir orientierten uns einerseits an den vielfältigen Strategien anderer Studien, insbesondere im Feld der partizipativen Forschung, entwickelten aber auch eine für unseren Kontext passende Auswertungsstrategie, die wir im Folgenden transparent machen, um damit auch zur Weiterentwicklung der AI-Methode, insbesondere im deutschen Sprachraum, beizutragen.

\subsubsection{Aufbereitung der Interviewdaten}

Die Sprachaufnahmen wurden vom Hochschulteam wörtlich transkribiert. Ein so entstandener, pseudonymisierter Reader wurde der Forschungsgemeinschaft in der Rohfassung zur Verfügung gestellt. Wir entschieden uns, von den Rohdaten auszugehen, anstatt das Material für die Auswertung vorab zu kategorisieren, um mit einer möglichst großen Offenheit in einem gemeinsamen Dialog nach Deutungs- und Interpretationsmöglichkeiten zu suchen (Cook 2012). Dafür war es notwendig, dass alle Mitglieder der Forschungsgemeinschaft, die an der Auswertung beteiligt waren, die Transkripte vollständig kannten und sich damit befassten. Mit der gemeinsamen Auswertung in der Forschungsgemeinschaft wollten wir die verschiedenen Sichtweisen zutage fördern und den Auswertungsprozess vielschichtig im Sinne partizipativer Forschung gestalten (Bergold \& Thomas 2012; von Unger 2014a).

Die Mitglieder der Forschungsgemeinschaft erhielten einen Leseauftrag, der die Auswertung vorbereitete: Sie sollten Textstellen markieren, die inhaltlich wichtig für die Beantwortung der handlungsleitenden Forschungsfrage waren (Wie kann die Zusammenarbeit im Netzwerk wieder in Schwung gebracht werden?). Zudem sollten Stellen gekennzeichnet werden, die bei den Mitforschenden eine emotionale Reaktion auslösten. Wir gehen davon aus, dass Betroffenheit auslösende Stellen eine hohe Relevanz besitzen (können) und ihnen in der Auswertung daher Beachtung geschenkt werden sollte. Textstellen, in denen Fragen offenblieben, waren ebenfalls zu markieren. Die Vorbereitung der Transkripte wurde innerhalb der Forschungsgemeinschaft aufgeteilt. Es stand jedoch allen offen, auch weitere Interviews zu bearbeiten.

\subsubsection{Dialogische Auswertung in der Forschungsgemeinschaft}

Der Auswertungsprozess begann mit einem zwei- und einem weiteren eintägigen Workshop. Die Grundlage bildete der von den Mitgliedern der Forschungsgemeinschaft nach dem oben genannten Leseauftrag bearbeitete Transkriptreader. Wir wollten in den Auswertungstreffen die verschiedenen Deutungs- und Interpretationsvarianten herausarbeiten und gleichzeitig das Material nach der Forschungsfrage und den Schwerpunktsetzungen der Mitforschenden filtern (Tuckermann \& Rüegg-Stürm 2010). 
Die gemeinsame Reflexion und Auswertung der AI-Interviews folgte diesen Teilschritten:

- Zunächst wurde der die Interviewpartner ${ }^{\star}$ in und die Atmosphäre im Interview selbst durch die Mitforschende, die das Interview geführt hatte, vorgestellt. Datenbögen und Memos dienten dem Gesprächseinstieg.

- Als zweiter Schritt stellte diejenige Person, die das entsprechende Interview vorbereitet hatte, die Textstellen vor, die sie gekennzeichnet hatte, und erklärte, was sie damit verband.

- Anschließend ergänzte in einem dritten Schritt die Gruppe ihre Erkenntnisse und Deutungen zum jeweils analysierten Interview und stieg in den Austausch hierüber ein.

- An einer Pinnwand trugen wir in einem vierten Schritt die Punkte zusammen, die aus Sicht der Beteiligten relevant für die Beantwortung der Forschungsfrage waren.

- Zuletzt sammelten wir Operationalisierungen, also erste Ideen dafür, wie sich das gemeinsam Herausgearbeitete konkret nutzen und was sich daraus lernen ließe.

Nach dem ersten Auswertungsworkshop und vor dem zweiten erhielten die Mitglieder der Forschungsgemeinschaft eine durch die beteiligten Wissenschaftlerinnen angefertigte Zusammenfassung der ersten vier analysierten Interviews. Diese Zusammenfassung des ersten Workshops diente dem Wiedereinstieg in die gemeinsame Analysetätigkeit. Im zweiten Auswertungsworkshop wurden die verbleibenden fünf Interviews nach dem oben vorgestellten Verfahren besprochen.

\subsubsection{Kategorisierung und Verschriftlichung der Ergebnisse}

Das Hochschulteam übernahm nach dieser gemeinsamen Arbeit den nächsten Überarbeitungsschritt. Es entwickelte entlang der Ergebnisse induktive Kategorien und ordnete diesen die besprochenen Textstellen aus dem Transkriptreader zu. Außerdem wurden weitere, zu der jeweiligen Kategorie passende Textstellen ausfindig gemacht und codiert. Dieser Schritt erfolgte in Anlehnung an inhaltsanalytische Verfahren und computergestützt mit der Analysesoftware MAXQDA (Kuckartz 2014). Um in der Forschungsgemeinschaft daran partizipativ weiterzuarbeiten, fertigte das Hochschulteam eine schriftliche Zusammenfassung der Kategorien an: Jede Kategorie wurde kurz definiert, dann wurde je Kategorie eine Liste mit den Themen aus den Auswertungsworkshops erstellt. Drei einschlägige Textstellen aus den Interviews verdeutlichten diese Zuordnung anhand des Materials, bevor alle unter die Kategorie fallenden Textstellen zusammenfassend als Fließtext 
dargestellt wurden. Die Operationalisierungen aus den Auswertungsworkshops wurden ebenfalls den Kategorien zugeordnet.

Erst nachdem die Forschungsgemeinschaft die Kategorien gemeinsam abgestimmt und Veränderungen beschlossen hatte, entstand ein gemeinsames Auswertungsdokument. Dieses Dokument diente der Forschungsgemeinschaft als Arbeitsgrundlage, um ergebnisorientierte Produkte und Veranstaltungen, unter anderem das bereits erwähnte Video, zu entwickeln. Wir zeigen im folgenden Abschnitt anhand der Entwicklung des Beratungsinstruments, wie wir unsere empirischen Ergebnisse aus der AI-Befragung nutzten und im Sinne der Destiny-Phase des 4D-Zyklus in Veränderungen für die Praxis überführten.

\subsubsection{Transfer der Ergebnisse in die Praxis am Beispiel des Beratungsinstruments}

Als ein Ergebnis der gemeinsamen Forschung entwickelten wir als Forschungsgemeinschaft das Beratungsinstrument „Standortanalyse für den Auf- und Ausbau integrierter kommunaler Strategien" sowie ein zugehöriges Begleitheft.

Die Standortanalyse eignet sich für die Beratung und Begleitung von Fachkräften, die eine IKS initiieren möchten oder die bereits damit begonnen haben und die Voraussetzungen für eine erfolgreiche Entwicklung überprüfen wollen. Wir wollen mit dem Instrument die Beratungsarbeit unterstützen und Fachkräften Mut machen, eine IKS auf- und auszubauen. Förderliche Bedingungen für den Aufbau einer IKS sind unseren Befragungsergebnissen nach:

- die soziale und gesundheitliche Lage der Bewohner*innenschaft zu kennen,

- sich Ziele zu setzen,

- den Sozialraum überschaubar zu halten,

- sich einen Überblick über die Netzwerkstrukturen zu verschaffen,

- Möglichkeiten für eine Koordinationsstelle im Sozialraum auszuloten, sich mit Anforderungen auseinanderzusetzen, die an Koordinierende gestellt werden, und ihre Potenziale zu kennen,

- die Verfügbarkeit von Ressourcen zu prüfen

- und sich ein Bild zu machen, wer vor Ort ist und sich einbringen will.

Die Ergebnisse leiten sich aus unserem gemeinsam erstellten Auswertungsdokument ab. „Zielorientiertes Arbeiten“ wurde von den von uns befragten Netzwerkmitgliedern als sehr wichtig eingeschätzt. Des Weiteren wünschten sich die Befragten, dass Ziele beibehalten werden, generell machbar sind und regelmäßig bilanziert werden. Außerdem erwarteten sie, sichtbare Ergebnisse zu erzielen, die sich mes- 
sen und dokumentieren lassen. Zwei Zitate aus den AI-Interviews unterstreichen die Bedeutung des zielorientierten Arbeitens für den Auf- und Ausbau von IKS.

Eine Fachkraft aus Rothenburgsort:

„Ich fand es wichtig, dass wir Ziele formuliert haben und mit Punkten bewertet haben, was uns wichtig ist und was wir priorisieren. Es ging darum, dass Angebote im Bereich Gesundheit ausgebaut werden und dass man sich besser vernetzt. Das fand ich gut, weil das sehr konkret und praktisch orientiert war und man auch wusste, woran man arbeitet. Es wurden die Ärmel hochgekrempelt und man hat von Anfang an gleich losgelegt."

Eine Mitarbeiterin des Bezirksamtes:

„Es gibt sichtbare Erfolge, wie den Stadtplan, der was zum Anfassen ist. Und dann denke ich, gibt es einen Output, der auf eine Art nicht direkt messbar ist. Der liegt darin, dass die Fachkräfte untereinander kommunizieren, besser miteinander agieren. Das hat natürlich auch Wirkung für die Bevölkerung im Stadtteil."

Wir schlussfolgerten aus unseren Ergebnissen, dass Entscheidungsträger*innen und Fachkräfte künftig dafür sensibilisiert werden müssen, wie sie den Auf- und Ausbau von IKS befördern können. Aus unserer Sicht sind dafür zwei Ansatzpunkte wesentlich: Erstens müssen die Adressat*innen oder Nutzenden einer IKS eine Stimme bekommen, gehört werden und ihre Vorschläge müssen in Abstimmung mit Entscheidungsträger*innen und Fachkräften, wenn möglich zeitnah, umgesetzt werden. Bottom-up und Top-down kommen auf diese Weise gelingend zusammen (Wihofszky 2015). Zweitens braucht es aus unserer Sicht Qualifizierungsangebote für die beteiligten Fachkräfte wie auch die Möglichkeit, sich durch Beratung und Coaching begleiten zu lassen. Dafür entwickelten wir das Instrument der Standortanalyse, das in der Beratung und Begleitung von Fachkräften eingesetzt werden kann.

Das Instrument orientiert sich, außer an unseren Forschungsergebnissen, am theoretischen Modell community readiness (Tri-Ethnic Center for Prevention Research 2014). Community readiness beschreibt Phasen, die durchlaufen werden, wenn Neuerungen in Stadtteilen eingeführt werden. Erst wenn eine gewisse „Handlungsbereitschaft“ oder „soziale Reife“ in einem Stadtteil erreicht ist, lohnt es sich, nächste Schritte der Planung und Umsetzung anzugehen (Brand et al. 2017; Reis-Klingspiegl 2009). Das Modell identifiziert dazu verschiedene Dimensionen, die die Einschätzung eines Sozialraums erlauben. Entsprechend unserer Forschungsergebnisse modifizierten wir die Dimensionen des Modells und entwickelten einen passenden Rahmen für unser Instrument. 
Anders als im Leitfaden zur Feststellung der readiness eines Sozialraums ist es uns wichtig, mit unserem Instrument Fachkräfte über einen längeren Zeitraum zu begleiten. Dazu geben wir nach einer Selbsteinschätzung Anregungen an die Hand, wie in Folgeterminen an den Themen weitergearbeitet werden kann. Auch hier flossen unsere AI-Forschungsergebnisse ein.

Das Instrument der Standortanalyse eignet sich für den Hamburger Kontext, da es die Bedingungen der Landesrahmenvereinbarung zur Umsetzung der nationalen Präventionsstrategie gemäß $\$ 20$ f SGB V im Land Hamburg zur Förderung von IKS aufgreift und sich in das Qualifizierungskonzept der KGC einbettet. Berater*innen der KGC und bezirklichen Multiplikator*innen soll in Hamburg mit der Standortanalyse ein Hilfsmittel an die Hand gegeben werden, um die Situation in einem Sozialraum gemeinsam mit Fachkräften zu reflektieren und einzuschätzen. Mögliche Adressat ${ }^{\star}$ innen für eine Beratung mit der Standortanalyse sind Koordinator ${ }^{\star}$ innen für Gesundheitsförderung in den Sozialräumen sowie Akteur*innen aus den Stadtteilen.

\section{Diskussion}

Für die Anwendung von AI ist es wesentlich, sich mit der Literatur der Begründer*innen der Methode auseinanderzusetzen (Cooperrider et al. 2008). Für die konkrete Vorbereitung und Durchführung unserer AI-Studie orientierten wir uns vorwiegend an Literatur aus dem anglofonen Raum, in der die Anwendung von AI im Rahmen von Forschungsprojekten thematisiert wird (Reed 2007; Trajkovski et al. 2013). Aber auch handlungsorientierte Literatur aus dem Bereich der Organisationsentwicklung erwies sich als eine relevante Quelle in der Umsetzung von AI (zur Bonsen \& Maleh 2012). In der Rezeption der Literatur sowie auch zahlreicher Studien, die im internationalen Kontext zur methodischen Anwendung von AI publiziert wurden, entwickelten und erprobten wir ein für unseren Kontext passendes Forschungsdesign, das wir mit seinen methodischen Schritten in diesem Beitrag dargestellt haben. In der nachfolgenden Diskussion reflektieren wir unser Vorgehen (5.1), beantworten die Frage, wie sich der Nutzen von AI aus verschiedenen Perspektiven der Forschungsgemeinschaft darstellt (5.2) und betrachten zuletzt Kritikpunkte und mögliche Limitationen der Anwendung der Methode (5.3). 


\subsection{Reflexion des methodischen Vorgehens}

Nach unseren Erfahrungen war es zunächst wichtig, sich mit der für AI besonderen Wertehaltung vertraut zu machen und sich damit auseinanderzusetzen. Dazu führten wir in unserer Forschungsgemeinschaft Schulungsworkshops durch, um diese positiv ausgerichtete Wertehaltung zu erlernen und einzuüben. Aus den Schulungsworkshops und den Erhebungen gewannen wir Erkenntnisse, wie sich die Wertehaltung von AI auswirkt. Die Mitglieder der Forschungsgemeinschaft meldeten zurück, dass die wertschätzende und erzählgenerierende Art zu fragen dazu führe, sich miteinander zu identifizieren und gut ins Gespräch zu kommen. Über Stärken zu sprechen, habe lebendige Erzählungen ermöglicht. Insgesamt empfand die Forschungsgemeinschaft das Interviewen nach AI als beziehungsstärkend. Diese Erfahrungen decken sich mit anderen AI-Studien (Bushe 2007; Dematteo \& Reeves 2011)

Die konsequent positive Frageweise von AI führte auch zu Schwierigkeiten. Manchen Interviewpartner*innen fiel es insgesamt eher schwer, auf das positiv Erlebte zu fokussieren. In der Praxis scheint es - verständlicherweise - eher üblich, auf auftretende Schwierigkeiten zu schauen und nach geeigneten Lösungen dafür zu suchen. Dafür war es nach unseren Erfahrungen wichtig, am Anfang der Interviews darauf hinzuweisen, dass auch Kritik ihren Platz haben kann. Wie eine Mitforschende sagte, sei auch ein „Ja, aber ..." möglich.

Die Frage nach Visionen und Zielen - in der AI-Terminologie oft als Wunderfrage in der Dream-Phase gestellt - brachte, wie dies auch Dematteo und Reeves (2011) konstatieren, den Effekt, Motivation (neu) zu entfachen und Handeln anzuregen. Die Interviewerinnen stellten fest, dass sie bzw. die Befragten durch diese Fragen auch bemerkten, dass und wie sie bereits an der Verwirklichung von Wünschen und Zielen arbeiteten. Den Begriff „Visionen“ empfanden einige der Mitforschenden als ungeeignet für den deutschen Sprachraum. Daher einigte sich die Forschungsgemeinschaft auf den Begriff „Ziele“ und formulierte eine Frage zur erwünschten Zukunft.

Insgesamt wurde das Interview als entzerrende Methode empfunden, die viele unterschiedliche Sichtweisen zutage fördert und neue Perspektiven ermöglicht. Auch wenn die Interviewerin zunächst das Gefühl habe, sich zu wiederholen, merke man jedoch, dass sich die Fragen in feinen, aber wichtigen Nuancen unterscheiden. Ein Mitglied der Forschungsgemeinschaft beschrieb dies als „Filetieren“, bei dem nach und nach neue Informationen und Aspekte ans Licht kämen.

In der Auswertung der AI-Interviews konnten wir, wie dies auch Carter, Cummings und Cooper (2007) berichten, nur auf überblickhafte Beschreibungen in der Literatur zurückgreifen. Dass hier methodische Vorgaben fehlten, an denen wir 
uns hätten orientieren können, war Chance und Herausforderung zugleich. Wir waren dadurch gezwungen, kreativ zu werden und die Daten in einem intensiven dialogischen Prozess zu interpretieren, der dennoch strukturiert und gegenstandsangemessen sein sollte. Die Herausforderung dabei war, die Auswertung für alle Beteiligten praktikabel zu gestalten. Dies wird auch zukünftig keine einfache Aufgabe sein, denn AI kann unserer Meinung nach auf unterschiedlichen Ebenen, für unterschiedliche Kontexte und verschiedene Nutzende fruchtbar gemacht werden.

\subsection{Perspektiven zum Nutzen der Methode}

In der Reflexion unseres Prozesses stellten wir als Forschungsgemeinschaft fest, dass sich der Gewinn durch die Anwendung von AI für uns unterschiedlich darstellt. Analog zu den beiden Zielebenen von partizipativer Forschung (von Unger 2014a) erkannten auch wir im Rückblick zwei Ebenen des Nutzens der Forschungsergebnisse.

Aus Sicht der HAG ermöglichte das Forschungsprojekt mit seinem partizipativen methodischen Vorgehen und der Anwendung von AI sowohl die Evaluation des Aufbauprozesses der IKS als auch die Aktivierung der zukünftigen Zusammenarbeit des Netzwerks im Rahmen der Regionalkonferenz (Göldner \& Hofrichter 2019). Durch die Anwendung von AI im Stadtteil Rothenburgsort, zu einem Zeitpunkt, als das bestehende Netzwerk ermüdet war und Veränderungen gegenüberstand, erwies sich die Methode für die Zusammenarbeit als höchst hilfreich. KEG ermöglichte für die Entwicklung von Gesundheitsförderung im Stadtteil einen lokalen Nutzen - Cook, Atkin und Wilcockson (2018) werten dies als ein entscheidendes Kriterium partizipativer Forschungsprojekte.

Darüber hinaus stellt AI aus Sicht der HAG auch eine geeignete Methode für die praktische Arbeit sowie für die fachliche Begleitung und Unterstützung der in der HAG angesiedelten KGC dar. Methodische Elemente und die Wertehaltung von AI können in die Angebote des praxisnahen Beratungs- und Qualifizierungskonzeptes der KGC integriert werden, unter anderem in Netzwerktreffen, Fach- und Erfahrungsaustausche sowie kollegiale und individuelle Beratungen.

Ähnlich wie die HAG äußerte sich auch die an der Forschungsgemeinschaft beteiligte Netzwerkkoordinatorin. Aus ihrer Sicht wirkte es ermutigend und motivierend, im Rahmen der AI-Interviews zurückzudenken und sich darauf zu besinnen, was bereits gut gelaufen war. Zudem trug das Projekt dazu bei, dass die am Netzwerk Beteiligten sahen und würdigten, dass und wie viel sie bereits initiiert und erreicht hatten. Vor allem der aus den Ergebnissen produzierte Film, in dem Originaltöne und Fotos zu hören und sehen sind, verdeutlichte die Arbeit und das Engagement des Netzwerks, auch gegenüber einer breiteren Öffentlichkeit. Dar- 
über hinaus wurden einige Ideen, die an die durch die AI-Befragung erkundeten Erfolgsfaktoren der Anfangszeit des Netzwerks anknüpften, im neu fusionierten Netzwerk angestoßen.

Aus der Perspektive der Hochschule gestaltete sich der AI-Prozess als innovativ, da eine im deutschen Sprachraum noch wenig bekannte Methode partizipativer Forschung erprobt und an den Kontext Stadtteil angepasst wurde. Der wesentliche Nutzen von AI besteht auch aus Sicht der wissenschaftlich Forschenden darin, Forschung und Intervention zu verbinden. In KEG war es uns möglich, nicht nur den Forschungsgegenstand „Zusammenarbeit im Rahmen von IKS“ zu erforschen und zu verstehen, sondern auch konkret Impulse zu geben, wie die Zusammenarbeit nach der Fusion des Netzwerks gestaltet werden kann. Nach Trajkovski und Kolleg*innen (2013) ist ein AI-Prozess nicht in sich geschlossen, sondern geht für die Praxis weiter. Für die Hochschulseite verbinden sich damit Herausforderungen, da Projekte in der Regel einen Abschluss haben. Als wichtiges Ergebnis des AI-Prozesses entwickelten wir als Forschungsgemeinschaft ein sowohl empirisch fundiertes als auch theoriegeleitetes Beratungsinstrument für die kommunale Praxis, das den Transfer der Ergebnisse in die Praxis und auch die Weiterentwicklung der Praxis sicherstellt.

\subsection{Kritische Einschätzung der Methode Al}

Wie wir beschrieben haben, ermöglicht die Anwendung von AI sowohl den Gewinn von praxisrelevanten Erkenntnissen als auch konkrete Veränderungen in der erforschten Praxis. Es bestätigten sich in unserer Fallstudie, wie auch in der Studie von Dematteo und Reeves (2011), die vielen positiven Wirkungen und der Nutzen der wertschätzenden Methode. Bezugnehmend auf Bushe (2007) und unsere eigenen Erfahrungen können diese mit dem generativen Potenzial, das der Anwendung von AI innewohnt, erklärt werden: AI fördert die Zuversicht und Hoffnung der Beteiligten, Lebensbedingungen zu verändern, stärkt soziale Beziehungen sowie auch personale Ressourcen wie zum Beispiel Selbstwirksamkeit und Kontrollüberzeugung.

Dennoch darf nicht außer Acht gelassen werden, dass sich diese Wirkungen von AI nur entfalten können, wenn strukturelle Rahmenbedingungen erfüllt sind. Wir teilen die Einschätzung von Bushe (2007), dass allein der Fokus auf das Positive und die Genese von Geschichten keine soziale Veränderung garantiert. Bezug nehmend auf Grant und Humphries (2006), die eine kritische Reflexion von Rahmenbedingungen fordern, sind auch wir überzeugt, dass in AI-Studien die Handlungsfähigkeit und -macht der Beteiligten sowie auch strukturelle Faktoren 
betrachtet werden müssen, die Handlungsmöglichkeiten einschränken. An dieser Stelle wollen wir nachdrücklich darauf hinweisen, dass eine Forschung mit AI in einem Klima der Unterdrückung ihr Potenzial nicht entfalten kann.

Ein weiterer, kritisch anzumerkender Punkt ist, dass AI die Fähigkeit erfordert, sich mündlich auszudrücken, und so bestimmte Personengruppen exkludiert, zum Beispiel Menschen mit Lernschwierigkeiten oder solche, die die Sprache, in der die Studie durchgeführt wird, nicht (in Gänze) beherrschen (Dittrich-Brauner et al. 2013).

Bei der konkreten Anwendung von AI in einem Netzwerk im Stadtteil ergeben sich weitere spezifische Limitationen. Ein Netzwerk ist in seiner Organisationsform fluid. Insbesondere das von uns untersuchte Netzwerk „Gesund aufwachsen in Rothenburgsort!" befand sich während des AI-Prozesses in einem Fusionsprozess. Deshalb konnten nicht alle wichtigen Akteur*innen für die Interviews erreicht werden, sodass unsere Ergebnisse nur einen Ausschnitt an Veränderungswünschen und -potenzialen im Stadtteil abbilden. Unsere Ergebnisse können auch nicht unhinterfragt auf andere Stadtteile und Kommunen übertragen werden, sondern mündeten, wie wir im Rahmen des Beitrags darstellten, in die Entwicklung eines Instrumentes, das die Reflexion bei der Einführung und dem Aufbau einer IKS fördern soll. Uns erscheint es auch in Bezug auf das Setting Stadtteil wichtig, neben den subjektiven Sichtweisen der Beteiligten auch die strukturellen Bedingungen im Auf- und Ausbau von IKS in Betracht zu ziehen. Die Partizipation - insbesondere der Adressat*innengruppen - stellt im Bereich der Gesundheitsförderung ein wesentliches Qualitätsmerkmal von Praxis dar (Wright et al. 2010). Deshalb sehen wir auch die geringe Einbindung von Bewohner*innen von Rothenburgsort in den AI-Prozess als eine Limitation unserer Forschung. Der Vorteil, Bewohner*innen stärker in den AI-Prozess einzubeziehen, läge darin, dass wichtige Sichtweisen auf den Stadtteil aus erster Hand zum Vorschein gebracht werden und zum Tragen kommen könnten.

\section{Ausblick}

AI eignet sich nach unserer Erfahrung als Methode für partizipative Forschungsprojekte. Die der Methode zugrunde liegende, konsequent wertschätzende Haltung ermöglicht es, mit einer ressourcenorientierten Perspektive den Forschungsgegenstand, in unserem Fall die Gesundheitsförderung in Stadtteilen und Kommunen, zu untersuchen und Veränderungen zu initiieren. Gerade im Bereich der kommunalen Gesundheitsförderung wäre es wichtig, Bewohner*innen in AI-Prozesse verstärkt einzubinden - zumal AI auch in Großgruppen methodisch sehr gut funktioniert 
(Dittrich-Brauner et al. 2013). Da AI als Forschungsmethode im deutschen Sprachraum noch nicht bekannt ist, wollen wir mit unserem Beitrag und der detaillierten Beschreibung unserer methodischen Schritte die Verbreitung und Rezeption von AI fördern. In zukünftigen AI-Projekten sollte aus unserer Sicht insbesondere auch der Frage nach Wirkungen verstärkt nachgegangen werden.

\section{Danksagung}

Wir bedanken uns bei allen mitwirkendenden Akteur*innen und Bewohner*innen des Stadtteils Rothenburgsort, die mit ihrem Engagement und ihrer Tatkraft zum Gelingen des Forschungsprojekts KEG beigetragen haben. KEG ist Teil des Forschungsverbundes PartKommPlus und wird von 2015 bis 2021 vom Bundesministerium für Bildung und Forschung (BMBF) mit dem Förderkennzeichen 01EL1823H gefördert. Unser Dank gilt auch allen am Verbund beteiligten Partner*innen. Der Verbund ist ein Projekt von PartNet, dem deutschen Netzwerk für Partizipative Gesundheitsforschung.

\section{Literatur}

Bergold, Jarg \& Thomas, Stefan (2012). Partizipative Forschungsmethoden: Ein methodischer Ansatz in Bewegung. Forum Qualitative Sozialforschung / Forum: Qualitative Social Research, 13(1), Art. 30, http://nbn-resolving.de/urn:nbn:de:0114-fqs1201302 [Zugriff: 30. Oktober 2018].

Böhme, Christa \& Reimann, Bettina (2018). Integrierte Strategien kommunaler Gesundheitsförderung: Rahmenbedingungen, Steuerung und Kooperation - Ergebnisse einer Akteursbefragung. Berlin: Deutsches Institut für Urbanistik gGmbH. http://edoc.difu. de/edoc.php?id=DIB7RTEK [Zugriff: 24. Oktober 2018].

Brand, Tilman; Gansefort, Dirk; Forberger, Sarah; Ubert, Tobias; Bröring, Eileen \& Zeeb, Hajo (2017). Bewegungsförderliche Kommune? Bedarfsermittlung und Entwicklung lokaler Steuerungskapazitäten. In Mario A. Pfannstiel, Axel Focke \& Harald Mehlich (Hrsg.), Management von Gesundheitsregionen II (S.51-60). Wiesbaden: Springer.

Bushe, Gervase R. (2007). Appreciative inquiry is not (just) about the positive. OD practitioner, 39(4), 30-35.

Carter, Bernie; Cummings, Julie \& Cooper, Lorraine (2007). An exploration of best practice in multi-agency working and the experiences of families of children with complex health needs. What works well and what needs to be done to improve practice for the future? Journal of Clinical Nursing, 16(3), 527-539. 
Cooperrider, David L.; Whitney, Diana \& Stavros, Jacqueline M. (2008). The appreciative inquiry handbook: for leaders of change. San Francisco: Berrett-Koehler Publishers.

Cook, Tina (2012). Where participatory approaches meet pragmatism in funded (health) research: the challenge of finding meaningful spaces. Forum Qualitative Sozialforschung / Forum: Qualitative Social Research, 13(1), Art. 18, http://dx.doi.org/10.17169/fqs-13.1.1783 [Zugriff: 12. November 2018].

Cook, Tina; Atkin, Helen \& Wilcockson, Jane (2018). Participatory research into inclusive practice: improving services for people with long term neurological conditions. Forum Qualitative Sozialforschung / Forum: Qualitative Social Research, 19(1), Art. 4, doi:10.17169/fqs-19.1.2667.

Dematteo, Dale \& Reeves, Scott (2011). A critical examination of the role of appreciative inquiry within an interprofessional education initiative. Journal of Interprofessional Care, 25(3), 203-208.

Dittrich-Brauner, Karin; Dittmann, Eberhard; Windisch, Carmen \& List, Volker (2013). Bewährte Großgruppenverfahren. In Karin Dittrich-Brauner, Eberhard Dittmann, Volker List, Carmen Windisch (Hrsg.), Interaktive Großgruppen: Change-Prozesse in Organisationen gestalten (S.27-127). Berlin, Heidelberg: Springer.

Farrell, Marie; Douglas, Dianna \& Siltanen, Susan (2003). Exploring and developing a college's community of interest: an appreciative inquiry. Journal of Professional Nursing, 19(6), 364-371.

Göldner, Josephine \& Hofrichter, Petra (2019). Gesundheitsförderung vor Ort gestalten Erfahrungen aus dem Stadtteil Rothenburgsort in Hamburg-Mitte. In Petra Kolip (Hrsg.), Praxishandbuch Qualitätsentwicklung und Evaluation in der Gesundheitsförderung (im Druck) (S. 164-178). Weinheim: Juventa.

Grant, Suzanne L. \& Humphries, Maria (2006). Critical evaluation of appreciative inquiry: bridging an apparent paradox. Action Research, 4(4), 401-418.

Helfferich, Cornelia (2011). Die Qualität qualitativer Daten: Manual für die Durchführung qualitativer Interviews. 4. Aufl. Wiesbaden: VS Verlag für Sozialwissenschaften / Springer Fachmedien.

Kuckartz, Udo (2014). Qualitative Inhaltsanalyse: Methoden, Praxis, Computerunterstützung. 2., durchges. Aufl. Weinheim: Beltz Juventa.

Laverack, Glenn \& Labonte, Ronald (2000). A planning framework for community empowerment goals within health promotion. Health policy and planning, 15(3), 255-262.

Ludema, James D. \& Fry, Ronald E. (2008). The practice of appreciative inquiry. In Peter Reason \& Hilary Bradbury (Hrsg.), The sage handbook of action research: participative inquiry and practice. Second Edition (S.280-296). London u. a.: SAGE Publications.

Nanz, Patrizia \& Fritsche, Miriam (2012). Handbuch Bürgerbeteiligung. Verfahren und Akteure, Chancen und Grenzen. Bonn: Bundeszentrale für politische Bildung. http:// www.bpb.de/system/files/dokument_pdf/Handbuch_Buergerbeteiligung.pdf [Zugriff: 15. August 2018].

Narimani, Petra (2014). Zustimmung als Prozess: Informiertes Einverständnis in der Praxisforschung mit von Ausweisung bedrohten Drogenabhängigen. In Hella von Unger, Petra Narimani \& Rosaline M'Bayo (Hrsg.), Forschungsethik in der qualitativen Forschung: Reflexivität, Perspektiven, Positionen (S.41-58). Wiesbaden: Springer VS.

Paige, Ciara; Peters, Ruth; Parkhurst, Malia; Beck, Leah L.; Hui, Brian; May, Vanessa T. \& Tanjasiri, Sora P. (2015). Enhancing community-based participatory research partners- 
hips through appreciative inquiry. Progress in community health partnerships: research, education, and action, 9(3), 457-463.

Quaintance, Jennifer L.; Arnold, Louise \& Thompson, George S. (2010). What students learn about professionalism from faculty stories: an „appreciative inquiry“ approach. Academic Medicine: Journal of the Association of American Medical Colleges, 85(1), 118-123.

Reed, Jan (2007). Appreciative inquiry: research for change. Thousand Oaks u.a.: SAGE Publications.

Reed, Jan; Pearson, Pauline; Douglas, Barbara; Swinburne, Stella, \& Wilding, Hellen (2002). Going home from hospital - an appreciative inquiry study. Health \& Social Care in the Community, 10(1), 36-45.

Reed, Jan; Richardson, Elsie; Marais, Sandra \& Moyle, Wendy (2008). Older people maintaining well-being: an international appreciative inquiry study. International Journal of Older People Nursing, 3(1), 68-75.

Reis-Klingspiegl, Karin (2009). Das steirische Netzwerk der Gesunden Gemeinden. Eine Entwicklungsgeschichte nach Ottawa. Prävention und Gesundheitsförderung, 3(4), 175-183.

Richter-Kornweitz, Antje \& Utermark, Kerstin (2013). Gesund aufwachsen für alle Kinder! Werkbuch Präventionskette: Herausforderungen und Chancen beim Aufbau von Präventionsketten in Kommunen. Hannover: Landesvereinigung für Gesundheit \& Akademie für Sozialmedizin Niedersachsen e. V. http://www.fruehehilfen.de/fileadmin/user_upload/ fruehehilfen.de/pdf/Publikation_Werkbuch_Praeventionskette.pdf [Zugriff: 18. September 2018].

Salyers, Michelle P.; Firmin, Ruth; Gearhart, Timothy; Avery, Elizabeth \& Frankel, Richard M. (2015). What we are like when we are at our best: appreciative stories of staff in a community mental health center. American journal of psychiatric rehabilitation, 18(3), 280-301.

Scerri, Anthony; Innes, Anthea, \& Scerri, Charles (2015). Discovering what works well: exploring quality dementia care in hospital wards using an appreciative inquiry approach. Journal of Clinical Nursing, 24(13-14), 1916-1925.

Springett, Jane; Wright, Michael T. \& Roche, Brenda (2011). Developing quality criteria for participatory health research: an agenda for action. WZB Discussion Paper, No. SP I 2011-302, Berlin: Wissenschaftszentrum Berlin für Sozialforschung (WZB).

Statistisches Amt für Hamburg und Schleswig-Holstein (2018a). Regionaldaten für Rothenburgsort. http://region.statistik-nord.de/detail/10000000000000/2/0/227688/ [Zugriff: 04. April 2019].

Statistisches Amt für Hamburg und Schleswig-Holstein (2018b). Regionaldaten für Hamburg. http://region.statistik-nord.de/detail/10000000000000/2/ [Zugriff: 04. April 2019].

Trajkovski, Suza; Schmied, Virginia; Vickers, Margaret \& Jackson, Debra (2013). Implementing the $4 \mathrm{D}$ cycle of appreciative inquiry in health care: a methodological review. Journal of Advanced Nursing, 69(6), 1224-1234.

Tuckermann, Harald \& Rüegg-Stürm, Johannes (2010). Researching practice and practicing research reflexively. Conceptualizing the relationship between research partners and researchers in longitudinal studies. Forum Qualitative Sozialforschung / Forum: Qualitative Social Research, 11(3), Art. 14, http://www.qualitative-research.net/index.php/fqs/ article/view/1540/3046 [Zugriff: 15. November 2018].

Tri-Ethnic Center for Prevention Research (Hrsg.) (2014). Community readiness for community change. Tri-ethnic center community readiness handbook. 2. Aufl. Fort Collins: Colorado State University. 
von Unger, Hella (2014a). Partizipative Forschung. Einführung in die Forschungspraxis. Wiesbaden: Springer VS.

von Unger, Hella (2014b). Forschungsethik in der qualitativen Forschung: Grundsätze, Debatten und offene Fragen. In Hella von Unger, Petra Narimani \& Rosaline M'Bayo (Hrsg.), Forschungsethik in der qualitativen Forschung: Reflexivität, Perspektiven, Positionen (S. 15-39). Wiesbaden: Springer VS.

von Unger, Hella \& Narimani, Petra (2012). Ethische Reflexivität im Forschungsprozess: Herausforderungen in der Partizipativen Forschung. WZB Discussion Paper, No. SP I 2012-304, Berlin: Wissenschaftszentrum Berlin für Sozialforschung (WZB).

WHO (1986). Ottawa-Charta zur Gesundheitsförderung. Genf: World Health Organization, www.euro.who.int/_data/assets/pdf_file/0006/129534/Ottawa_Charter_G.pdf [Zugriff: 15. August 2018].

Wihofszky, Petra (2015). Ein mittlerer Weg zwischen Top-down und Bottom-up in der Gesundheitsförderung. Impu!se für Gesundheitsförderung, (88), 5-6.

Wright, Michael T. (2013). Was ist Partizipative Gesundheitsforschung? Positionspapier der International Collaboration for Participatory Health Research. Prävention und Gesundheitsförderung, 8(3), 122-131.

Wright, Michael T. (2016). Partizipative Gesundheitsforschung. In Bundeszentrale für gesundheitliche Aufklärung (BZgA) (Hrsg.), Leitbegriffe der Gesundheitsförderung, https:// www.leitbegriffe.bzga.de/alphabetisches-verzeichnis/partizipative-gesundheitsforschung/ [Zugriff: 11. März 2019].

Wright, Michael T.; von Unger, Hella \& Block, Martina (2010). Partizipation der Zielgruppe in der Gesundheitsförderung und Prävention. In Michael T. Wright (Hrsg.), Partizipative Qualitätsentwicklung in der Gesundheitsförderung und Prävention (S. 35-52). Bern: Verlag Hans Huber.

zur Bonsen, Matthias \& Maleh, Carole (2012). Appreciative Inquiry (AI): Der Weg zu Spitzenleistungen. Eine Einführung für Anwender, Entscheider und Berater. 2. Aufl. Weinheim.

Open Access Dieses Kapitel wird unter der Creative Commons Namensnennung 4.0 International Lizenz (http://creativecommons.org/licenses/by/4.0/deed.de) veröffentlicht, welche die Nutzung, Vervielfältigung, Bearbeitung, Verbreitung und Wiedergabe in jeglichem Medium und Format erlaubt, sofern Sie den/die ursprünglichen Autor(en) und die Quelle ordnungsgemäß nennen, einen Link zur Creative Commons Lizenz beifügen und angeben, ob Änderungen vorgenommen wurden.

Die in diesem Kapitel enthaltenen Bilder und sonstiges Drittmaterial unterliegen ebenfalls der genannten Creative Commons Lizenz, sofern sich aus der Abbildungslegende nichts anderes ergibt. Sofern das betreffende Material nicht unter der genannten Creative Commons Lizenz steht und die betreffende Handlung nicht nach gesetzlichen Vorschriften erlaubt ist, ist für die oben aufgeführten Weiterverwendungen des Materials die Einwilligung des jeweiligen Rechteinhabers einzuholen.

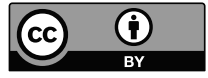

